Nervenarzt 2020 $911: 777-778$

https://doi.org/10.1007/s00115-020-00964-3

(c) Springer Medizin Verlag GmbH, ein Teil von Springer Nature 2020

\section{Georg Schomerus' ${ }^{1}$ Steffi Riedel-Heller ${ }^{2}$}

${ }^{1}$ Klinik und Poliklinik für Psychiatrie und Psychothreapie, Universitätsklinikum Leipzig, Leipzig, Deutschland

${ }^{2}$ Institut für Sozialmedizin, Arbeitsmedizin und Public Health, Universität Leipzig, Medizinische Fakultät, Leipzig, Deutschland

\title{
Das Stigma psychischer Krankheit im Fokus
}

In Zeiten, in denen die COVID-19-Pandemie unser gesellschaftliches Miteinander auf vielen Ebenen einschneidend verändert, wird einmal mehr deutlich, wie stark dieses Miteinander auch unseren Umgang mit psychischer Krankheit prägt. Gesellschaftliche Prozesse haben Einfluss darauf, wie gut oder schlecht man mit einer psychischen Krankheit leben kann. So ist auch das Stigma psychischer Krankheit nicht in erster Linie ein psychiatrisches Problem, sondern ein gesellschaftliches. Schließlich ist Stigma kein Krankheitssymptom, sondern Ausdruck des gesellschaftlichen Umgangs mit psychischer Krankheit. Stigmatisierung findet mehr oder weniger stark in Abhängigkeit davon statt, wie gut oder wie schlecht wir als Gesellschaft mit psychischer Krankheit umgehen können.

\section{》) Stigma beeinträchtigt den Verlauf psychischer Krankheiten}

Trotzdem ist die Psychiatrie eng mit dem Phänomen Stigma verbunden: zum einen, weil durch die psychiatrische Diagnose und Behandlung ein Labeling stattfindet, das zum Prozess der Stigmatisierung beitragen kann [2], zum anderen, weil öffentliches Stigma, Selbststigma und strukturelle Diskriminierung das Befinden unserer Patientinnen und Patienten beeinträchtigen, den Verlauf psychiatrischer Krankheiten negativ beeinflussen und vielfach auch der Inanspruchnahme professioneller Hilfe im Weg stehen.
In diesem Themenheft des Nervenarztes widmen sich 4 Arbeiten verschiedenen Aspekten der Stigmatisierung von Menschen mit psychischen Krankheiten und zeigen sowohl neue Richtungen wie auch praktische Implikationen der Stigmaforschung auf. Die 1. Arbeit untersucht den Zusammenhang zwischen Suizidalität und der Stigmatisierung suizidalen Verhaltens. Erst kürzlich ist insbesondere das Risiko poststationärer Suizide wieder in den Fokus gelangt [5]. Oexle et al. analysieren in einer Übersicht, die mit der medizinischen Literatur der Antike beginnt, den komplexen Zusammenhang zwischen Suizidstigma und Suizidprävention. Im 2. Beitrag des Themenheftes zeigen Speerforck und Schomerus auf, dass das Konzept der sozialen Milieus hilfreich sein kann, um Bevölkerungsgruppen zu definieren, bei denen einerseits stigmatisierende Einstellungen gegenüber psychischer Krankheit besonders prävalent sind und die andererseits besonders schlecht von psychiatrischen Versorgungsangeboten erreicht werden. Die Arbeit trägt damit auch der Tatsache Rechnung, dass die Unter- und Fehlversorgung von Menschen mit psychischen Krankheiten immer noch unzureichend verstanden ist [1].

Die Stigmaforschung hat sich in den letzten Jahren ausführlich damit befasst, ob sich bestimmte ätiologische Vorstellungen von psychischer Krankheit günstig auf die Stigmatisierung auswirken oder nicht. Die zunächst weit verbreitete Hoffnung, biologische Krankheitskonzepte könnten entstigmatisierend wirken, darf als widerlegt gelten [4]. Weit weniger untersucht ist jedoch 
$(2$

die Frage, ob auch Behandlungskonzepte zur Entstigmatisierung beitragen können.

\section{》) Ein konstruktiver Umgang mit psychischer Krankheit kann zur Entstigmatisierung beitragen}

$\mathrm{Ob}$ gute, qualitätsgesicherte psychiatrische Behandlung zur Entstigmatisierung von Personen mit psychischen Erkrankungen beitragen kann, zeigt die Übersichtsarbeit von Gaebel und Stricker. Wenn das Stigma psychischer Krankheit als Ausdruck eines dysfunktionalen gesellschaftlichen Umgangs mit psychischer Krankheit angesehen wird, dann ist ein wichtiger Beitrag der Psychiatrie zur Entstigmatisierung tatsächlich die Vermittlung eines leitliniengerechten, wertschätzenden, ermutigenden und damit konstruktiven Umgangs mit psychischer Krankheit.

Auch Schlier et al. untersuchten in einer experimentellen Studie, die als Originalarbeit dem Leitthema beigefügt ist, ob medikamenten- oder psychotherapiegestützte Behandlungskonzepte bei Schizophrenie die Stigmatisierung einer betroffenen Person und die Erwartungen hinsichtlich einer Genesung beeinflussen.

Die Entstigmatisierung psychischer Krankheit ist eine Aufgabe, die viele Akteure fordert, nicht zuletzt den Einsatz von Menschen mit Krankheitserfahrung [3]. Die Beiträge dieses Themenschwerpunkts zeigen auf, dass die Aufgabe noch nicht gelöst ist und dass auch die Psychiatrie in Wissenschaft und Praxis hier ihren Beitrag leisten kann.

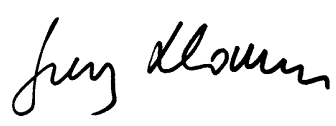

Prof. Dr. Georg Schomerus

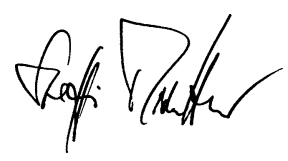

Prof. Dr. Steffi Riedel-Heller

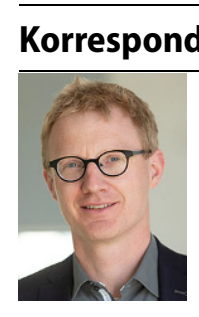

Aktuelle Informationen
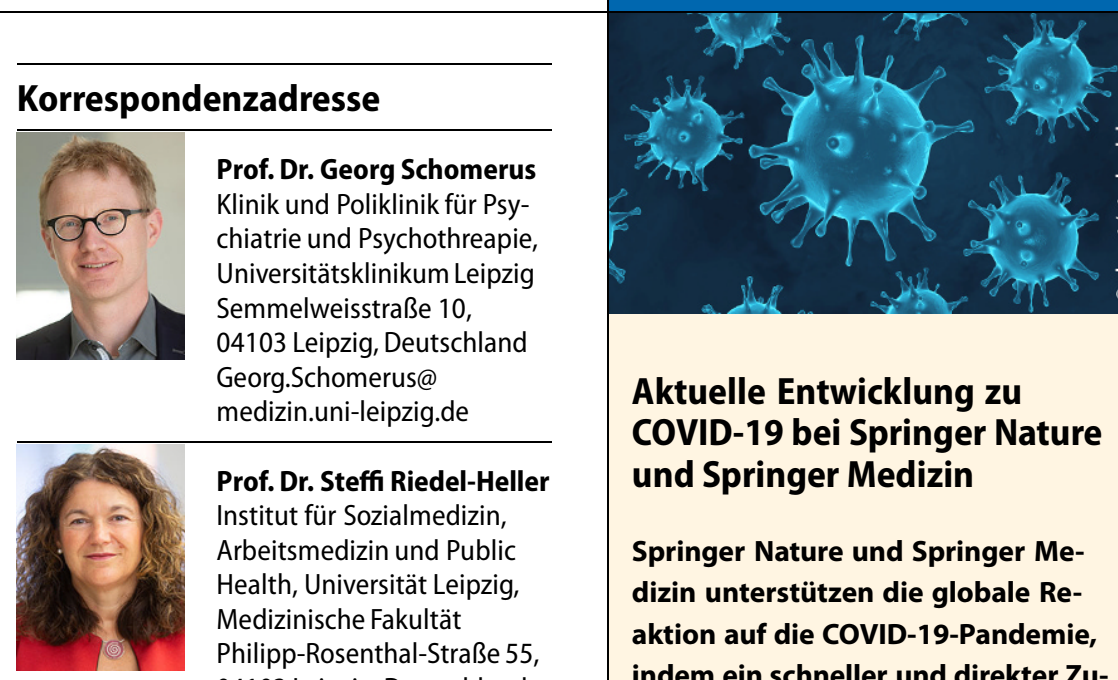

Prof. Dr. Georg Schomerus Klinik und Poliklinik für Psychiatrie und Psychothreapie, Universitätsklinikum Leipzig Semmelweisstraße 10, 04103 Leipzig, Deutschland Georg.Schomerus@ medizin.uni-leipzig.de

Prof. Dr. Steffi Riedel-Heller Institut für Sozialmedizin, Arbeitsmedizin und Public Health, Universität Leipzig, Medizinische Fakultät Philipp-Rosenthal-Straße 55, 04103 Leipzig, Deutschland Steffi.Riedel-Heller@medizin.uni-leipzig.de

Interessenkonflikt. G. Schomerus und S. RiedelHeller geben an, dass kein Interessenkonflikt besteht.

\section{Literatur}

1. Fegert JM, Baumeister $H$, Brieger $P$ et al (2019) Greifswalder Erklärung zur gesellschaftlichen Bedeutung des Bereichs psychische Gesundheit in der Gesundheitforschung - „Lost in Translation?". Psychiatr Prax 46:70-72

2. LinkBG, Phelan JC (2001) Conceptualizing stigma. Annu Rev Sociol 27:363-385

3. Rüsch N, OexleN, Reichhardt Let al (2019) In Würde zu sich stehen - Konzept und Wirksamkeit eines peer-geleiteten Programms zu Offenlegung und Stigmabewältigung. Psychiatr Prax 46:97-102

4. Schomerus $G$, Matschinger $H$, Angermeyer MC (2014) Causal beliefs of the public and social acceptance of persons with mental illness: a comparative analysis of schizophrenia, depression and alcohol dependence. Psychol Med 44:303-314

5. SpießI H (2019) Mehr Fokus auf poststationäre Suizide! Psychiatr Prax 46:67-69
Springer Nature und Springer Medizin unterstützen die globale Reaktion auf die COVID-19-Pandemie, indem ein schneller und direkter $\mathrm{Zu}$ gang zu den neuesten verfügbaren Forschungsergebnissen und Daten ermöglicht wird.

Auf der Homepage SpringerMedizin.de finden Sie ein immer aktuelles Dossier mit Beiträgen, Forschungsarbeiten und Ergebnissen zu SARS-CoV-2 sowie relevanten Links.

Darin z.B. auch die kürzlich publizierte

Empfehlung von DIVI, DGIIN, DGAI und DGP zur Intensivtherapie von Patienten mit COVID-19.

Springer Nature arbeitet mit globalen Organisationen zusammen, und verlinkt über SpringerNature.com/de auf eine eigene Landingpage mit einer Vielzahl an Information sowie freiem Zugriff auf die COVID-19-Contentplattformen von Nature Research, BioMed Central (BMC) und Springer.

Das Dossier zu Coronavirus / Covid-19 von Springer Medizin finden Sie hier: www.springermedizin.de/covid-19 